\title{
Assessment of knowledge on pulse oximetry screening for critical congenital heart disease among nursing officers attached to postnatal units of a teaching hospital in Sri Lanka
}

\author{
Kanishka Sampath Thilakarathna ${ }^{1}$, Sugandi Savithri ${ }^{1}$, Aanjeli Wimalasiri ${ }^{1}$, Niroshini Ekneligoda ${ }^{1}$, \\ *Medha Weerasekera ${ }^{2}$
}

Sri Lanka Journal of Child Health, 2019; 48(2): 146-151

\begin{abstract}
Introduction: Critical congenital heart disease (CCHD) refers to any severe cardiac anomaly existing since birth and requiring surgical or catheter-based intervention during infancy
\end{abstract}

Objective: To assess knowledge on pulse oximetry (POS) protocol and its interpretation among nursing officers attached to postnatal wards of Sri Jayewardenepura General Hospital.

Method: A descriptive cross-sectional study was carried out to assess the nursing officers' knowledge on POS. Data were collected from the nursing officers in the postnatal wards who had working experience of one month or more by using an interviewer administered questionnaire. According to the responses, results were graded as unacceptable, acceptable and satisfactory.

Results: Fifty three female nurses working in the postnatal wards participated in the study. The average working experience was 72 months. The average score obtained was 6.4 (SD 1.63) out of a total of 9 . Ninety one percent scored above 5, 98\% knew the correct sites of saturation measurements, $91 \%$ were aware about the purpose of neonatal POS and $66 \%$ knew the optimal time of performing the test. Seventy percent knew the test is positive if the $\mathrm{SpO}_{2}$ difference between limbs is more than 3, but only $43 \%$ knew the test is positive if $\mathrm{SpO}_{2}$ is $<90 \%$ in any limb. Further, only $52 \%$ were aware of interpreting inconclusive test results $\left(\mathrm{SpO}_{2} 90\right.$ $94 \%)$. There was no significant difference $(\mathrm{p}<0.05)$ in the knowledge according to working experience.

\footnotetext{
${ }_{1}^{1}$ Regristrar in Paediatrics, Neonatal Intensive Care Unit, ${ }^{2}$ Consultant Neonatologist, Sri Jayewardenepura General Hospital, Nugegoda, Sri Lanka

*Correspondence:medhaweerasekera@gmail.com

(iD https://orcid.org/0000-0001-5065-1889

(Received on 01 July 2018: Accepted after revision on 17 August 2018)

The authors declare that there are no conflicts of interest Personal funding was used for the project.
} Commons Attribution CC-BY License
Conclusions: More than $90 \%$ of nursing officers had acceptable or satisfactory knowledge of POS. More than $90 \%$ knew the purpose of screening and the appropriate limbs to check saturations. Only $66 \%$ knew the optimal time to perform the study. Lag of knowledge was seen in interpretation of positive as well as inconclusive results.

DOI: http://dx.doi.org/10.4038/sljch.v48i2.8709

(Key words: Critical congenital heart disease, pulse oximetry screening, knowledge, nursing officers)

\section{Introduction}

Critical congenital heart disease (CCHD) refers to any severe cardiac anomaly existing since birth and requiring surgical or catheter-based intervention during infancy ${ }^{1}$. It consists of cyanotic cardiac defects and left sided obstructive lesions. Congenital heart disease (CHD) occurs in approximately $1 \%$ of live births ${ }^{1}$. About one quarter of children with CHD have critical defects ${ }^{2}$. Some babies are diagnosed prenatally or are symptomatic at birth. However, cyanosis is not detected by the human eye until the oxygen saturation reduces to approximately $80 \%{ }^{3}$. Thus, the remaining cases are often diagnosed only after hospital discharge ${ }^{4}$. These babies appear healthy at birth but succumb to heart failure or ductal closure during the neonatal period. Delayed diagnosis increases the mortality and morbidity of these life-threatening conditions.

As a measure to improve the detection of newborns with CCHD, universal pulse oximetry screening (POS) was introduced in 2011 in the United States for all babies before discharge from hospital ${ }^{5}$. In Sri Lanka, this intervention was introduced by the Ministry of Health from January 2017 for all babies born in government hospitals by monitoring preductal (right upper limb) and post-ductal (either lower limb) saturations before discharge from hospital ${ }^{6}$. POS is painless, non-invasive and widely used $^{7}$. The test is inexpensive so that screening for CCHD is cost effective ${ }^{8}$. Guidelines and protocols have been issued by the Ministry of Health for screening of $\mathrm{CCHD}^{6}$. The guidelines include information on how to perform the test (equipment used, probe placement), who will do the test and when, informing parents, and a simplified algorithm (Figure 1) to interpret the results. This screening 
practice is carried out primarily by nursing officers working in postnatal wards. With the introduction of universal pulse oximetry screening of newborns in Sri Lanka, it is imperative that continuous precision in screening is maintained. Therefore, the knowledge of postnatal nurses, of the procedure, protocol and interpretation of results is of paramount importance.

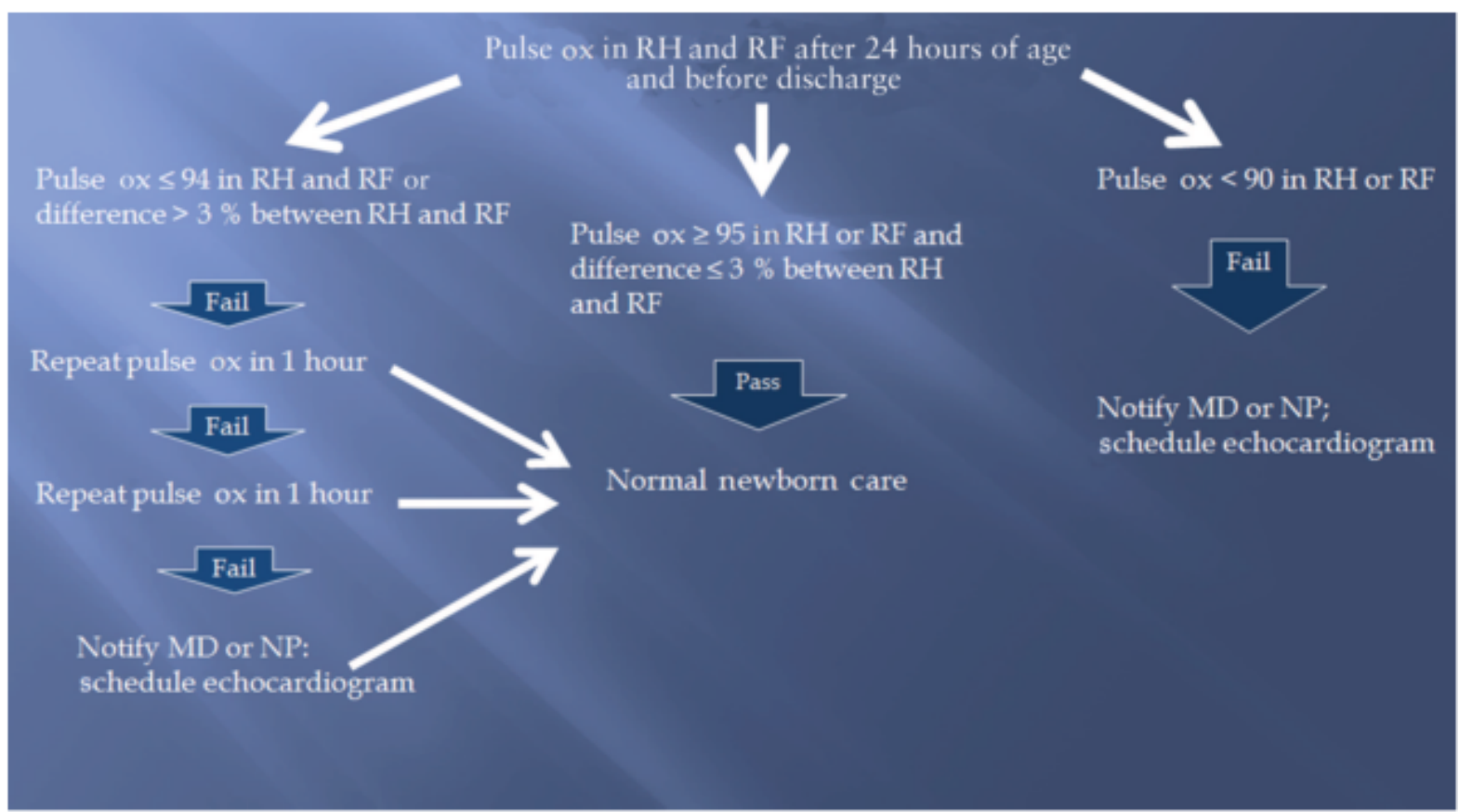

Figure 1: Protocol for pulse oximetry screening of newborns to detect critical congenital heart disease

Introduction of routine POS has led to early detection of CCHD which has led to timely intervention and reduction in morbidity and mortality. Though POS was introduced since January 2017 in Sri Lanka, no studies have been carried out to date to evaluate the nursing officer's knowledge on the methodology or interpretation. Such a study will help to identify areas of lag in knowledge and also quality of practice.

This study focused on assessing the knowledge and understanding of the screening protocol and procedure by nursing officers, who routinely perform this screening in postnatal wards. Furthermore, while collecting data we planned to address areas of lag in knowledge and misunderstandings of protocol.

\section{Objective}

To assess knowledge on POS protocol and its interpretation among nursing officers attached to postnatal wards of Sri Jayewardenepura General Hospital.

\section{Method}

Design: Descriptive cross sectional study

Setting and population: All nursing officers who give care for postnatal patients for one month or more. Pupil nurses were excluded.
Study instrument: An interviewer administered questionnaire, based on the current guideline was used to assess participants' knowledge. The questionnaire included 9 questions covering key aspects of POS - purpose, timing, measuring sites and interpretation of the results. Answers compatible with the guideline were taken as correct and other answers were marked as incorrect. Each correct answer was given one mark and results were graded depending on the total score.

Analysis: Data was analysed using SPSS 20.

Participants' knowledge was assessed on each component of the questionnaire and were graded according to the total score achieved.

0-5 = Unacceptable knowledge

6-7 = Acceptable knowledge

$>7=$ Satisfactory knowledge

Ethical issues: The Ethics Review Committee of the Sri Jayewardenepura General Hospital approved the study prior to its initiation. After data collection all participants were educated on their deficiencies in knowledge with regard to POS.

\section{Results}

Fifty-three nursing officers working in 4 postnatal units of Sri Jayewardenepura General Hospital were included in the study and all of them were females. Their work experience in the current unit ranged from 1 to 403 months with a mean experience of 72 months (SD 90.47). 
The average total score obtained was 6.43 (SD 1.63) with 2 participants $(3.8 \%)$ obtaining the lowest score of 3 and 6 participants $(11.3 \%)$ scoring the maximum score of 9 . Five $(9.4 \%)$ of the 53 participants had unacceptable knowledge, while 48
(90.6\%) scored above 5 (cumulative acceptable and satisfactory knowledge), with 17 (32\%) having a satisfactory knowledge. Grading of knowledge according to the correct answers given is depicted in Table 1.

Table 1: Grading of knowledge based on correct answers given regarding pulse oximetry

\begin{tabular}{|l|c|}
\hline $\begin{array}{c}\text { Grading of knowledge based on correct answers } \\
\text { (from a total score of 9) }\end{array}$ & Number (\%) of participants \\
\hline Unacceptable (0-5) & $05(09.4)$ \\
\hline Acceptable (6-7) & $31(58.5)$ \\
\hline Satisfactory $(>7)$ & $17(32.1)$ \\
\hline
\end{tabular}

Majority of the participants $(90.6 \%)$ were aware of the purpose of neonatal POS. Thirty-five $(66 \%)$ nursing officers knew the optimal time to perform the test. Almost all the participants (98.1\%) knew the correct sites of pre-ductal and post-ductal saturation measurements. Forty-six out of 53 $(86.8 \%)$ knew that screening is negative if both limb
$\mathrm{SpO}_{2}$ are above $95 \%$ and $37(69.8 \%)$ knew that $\mathrm{SpO}_{2}$ difference should be less than 4 for a negative screening test. Only $23(43.4 \%)$ knew that the test is positive if $\mathrm{SpO}_{2}$ is below $90 \%$ in any limb. Summary of all the correct responses of each component of the questionnaire is shown in Table 2.

Table 2: Number/percentage of correct responses in each component $(n=53)$

\begin{tabular}{|l|c|}
\hline Component & Number (\%) of participants who correctly answered \\
\hline Purpose of the pulse oximetry (POS) & $48(90.6)$ \\
\hline Timing of screening & $35(66.0)$ \\
\hline Site of pre-ductal saturation & $52(98.1)$ \\
\hline Site of post -ductal saturation & $52(98.1)$ \\
\hline POS is negative if $\mathrm{SpO}_{2}>95 \%$ & $46(86.8)$ \\
\hline POS is negative if $\mathrm{SpO}_{2}$ difference is $<4$ & $37(69.8)$ \\
\hline Screening is positive if $\mathrm{SpO}_{2}<90 \%$ in any limb & $23(43.4)$ \\
\hline $\begin{array}{l}\text { Number of repeated measurements to be taken if } \\
\text { SPO2 is } 90 \%-94 \% \text { or difference }>3\end{array}$ & $28(52.8)$ \\
\hline Time gap between repeated readings & $21(39.6)$ \\
\hline
\end{tabular}

Of the 53 nurses, $28(52.8 \%)$ knew that the procedure should be repeated twice in situations where inconclusive $\mathrm{SpO}_{2}$ results were obtained (i.e. $\mathrm{SpO}_{2}$ 90\%-94\%), and only 21 (39.6\%) knew the time gap should be one hour in such situations. One of the officers who scored a total of 3 was unaware about the correct $\mathrm{SpO}_{2}$ measuring sites including the rest of the procedure. She had a work experience of 1 month. However, the knowledge of nursing officers who had a working experience below the average ( 72 months or less) was not significantly different when compared with nursing officers with an experience above 72 months ( $\mathrm{p}>0.05)$.

\section{Discussion}

By the end of 1960, newborn screening for metabolic and genetic disorders became standard practice across the United States of America (USA) ${ }^{9}$. However, this practice evoked great interest and controversy. Therefore, in 1968 WHO released a document describing ten important criteria to be fulfilled before a disease is considered for universal screening ${ }^{10}$. This report is currently used as the gold standard before choosing a disease for universal screening.
CHD is the commonest birth anomaly and is a major cause of infant mortality ${ }^{11,12}$. It is responsible for 6$10 \%$ of infant mortality and $30-50 \%$ of mortality from congenital abnormalities ${ }^{13}$. Over time, CHD prevalence has increased from 0.6 per 1000 live births in the 1930s to 9 per 1000 live births in the $1990 \mathrm{~s}^{14}$. CHD birth prevalence varies geographically. Each year there are over one million births with CHD worldwide ${ }^{14}$.

CCHD prevalence in the USA is 2.9 per 1000 live births ${ }^{15}$. Survival of these newborns depend on early detection and intervention. Studies show that before POS was introduced, about $40 \%$ of newborns with CCHD were discharged home undiagnosed in the $\mathrm{USA}^{2}$. These estimates emphasize the need to screen newborns for CCHD. Although universal CCHD screening is carried out in the USA since $2011^{16,17}$, only a few European countries include POS in their universal screening panel ${ }^{18}$. In Sri Lanka, it was implemented as a national programme since $2017^{6}$.

In the United Kingdom (UK) $30 \%$ of potentially life threatening cardiovascular malformations in infants are diagnosed after discharge and 5\% are diagnosed 
after death ${ }^{19}$. Delayed or missed CCHD diagnosis accounts for 0.14 of deaths per 1000 live births in the $\mathrm{USA}^{20}$. Further, $10 \%$ of infants with CCHD die before a diagnosis could be made ${ }^{21}$. This diagnosis gap may be even higher in developing countries though sufficient data are not available. Studies show that POS improves early CCHD detection thus reducing the diagnosis gap ${ }^{22}$. POS has a specificity rate greater than $99 \%$ and a false-positive rate (FPR) less than $1 \%{ }^{8,15}$. Studies also reveal that FPR drops to $0.035 \%$ if screening is done 24 hours later. It is also estimated that POS can detect around $60 \%$ of infants born with undiagnosed CCHD. Furthermore, about $50 \%$ of false positives detected by POS are due to other non-cardiac pathology such as respiratory disorders, infections, pulmonary hypertension and hypothermia. This in turn is an additional advantage of doing $\operatorname{POS}^{17}$. The main limitation of POS is the low sensitivity rate estimated by most studies, ranging from $62 \%$ to $78 \%$ due to human error in following protocol, and misinterpretation of algorithm ${ }^{22}$.

In our study, knowledge regarding the key aspects of the procedure such as purpose and site of POS (90.6\% and $98.1 \%$ respectively) was satisfactory. However, only $66 \%$ knew the correct time to perform the test. Since the sensitivity of the test is dependent on correct timing, it is important for all the officers to have a clear idea on it. Having this insight will also reduce false positive interpretations since in the first 24 hours of life even a healthy neonate may have a $\mathrm{SpO}_{2}<90 \%$.

Amongst the participants, 90.6\% had acceptable or satisfactory level of knowledge. Although nearly $90 \%$ of the study population gave correct answers for more than half of the questions, only a minority $(11.3 \%)$ had good understanding on the entire procedure. For a more effective national programme it is important that the performers have a sound knowledge on all aspects of the procedure. Therefore, programmes to enhance the knowledge and clear doubts on the procedure would be beneficial. However, despite satisfactory knowledge on performing POS, there was a lag in knowledge related to interpretation of positive as well as inconclusive results. Although $86.8 \%$ knew the procedure is considered negative if the $\mathrm{SpO}_{2}$ value was more than $95 \%$ and $69 \%$ knew POS is negative if SPO2 difference between limbs is less than 4, only a mere $43.4 \%$ knew the procedure is positive if $\mathrm{SpO}_{2}$ is $<90 \%$ in any limb. Additionally, the participants did not have a clear understanding on steps to be taken with inconclusive results $\left(\mathrm{SPO}_{2}\right.$ between 90 94\%). Furthermore, only 52.8\% knew the test should be repeated twice in such instances, while only $39.6 \%$ knew 2 repeated measurements should be done in one hour time gaps. Thus, the need to focus on algorithm interpretation is further highlighted.
Though there was a wide variation in working experience, its effect on knowledge on POS was insignificant. Rather than the working experience, the knowledge and the practical aspects of the procedure may depend on the frequency of performing the procedure. Therefore, work experience per se may not have an impact on the knowledge on POS. The validity and the sensitivity of the screening procedure are dependent on the technique used. Though the knowledge on newborn POS is satisfactory in this study population, an assessment on the techniques adapted in performing the procedure was not looked into. It will be beneficial to look into it in future studies in order to increase the sensitivity of the procedure. Increasing education opportunities for staff on electronic interpretation of data, frequent quality improving by clinical audits, can help in identifying and correcting these issues. A large-scale study involving staff and parents to check their knowledge and perception regarding POS will help to establish more sustainable quality and cost-effective POS.

\section{Conclusions}

More than $90 \%$ of nursing officers had acceptable or satisfactory knowledge of POS. More than 98\% knew the purpose of screening and the appropriate limbs to check saturations. Only $66 \%$ knew the optimum time to perform the study. Lag of knowledge was seen in interpretation of positive as well as inconclusive results.

\section{References}

1. Oster M. Newborn screening for critical congenital heart disease using pulse oximetry. Available from:

https://www.uptodate.com/contents/newbo rn-screening-for-critical-congenital-heartdisease-using-pulse-oximetry

2. Oster ME, Lee KA, Honein MA, RiehleColarusso T, Shin M, Correa A. Temporal trends in survival among infants with critical congenital heart defects. Pediatrics 2013; 131(5): e1502e1508. https://doi.org/10.1542/peds.2012-3435 PMid: 23610203 PMCid: PMC4471949

3. Hokanson JS. Pulse oximetry screening for unrecognized congenital heart disease in neonates. Neonatology Today 2010; 5(12): 1-6.

4. Wren C, Richmond S, Donaldson L. Presentation of congenital heart disease in infancy: implications for routine examination. Archives of Disease in 
Childhood-Fetal and Neonatal Edition 1999; 80(1), F49-F53.

https://doi.org/10.1136/fn.80.1.F49 PMid: 10325813 PMCid: PMC1720871

5. Kumar P. Universal pulse oximetry screening for early detection of critical congenital heart disease. Clinical Medicine Insights: Pediatrics 2016; 10: 35-41.

https://doi.org/10.4137/CMPed.S33086

6. Intranatal and Newborn Care - Circular on Newborn screening to detect critical congenital heart disease. Family Health Bureau, Sri Lanka.

7. Ryan DJ, Mikula EB, Germana S, Silva SG, Derouin A. Screening for critical congenital heart disease in newborns using pulse oximetry: evaluation of nurses' knowledge and adherence. Advances in Neonatal Care 2014; 14(2): 119-28. https://doi.org/10.1097/ANC.0000000000 000047

PMid: 24675632

8. Granelli ADW, Wennergren M, Sandberg $\mathrm{K}$, Mellander M, Bejlum C, Inganäs L, et al. Impact of pulse oximetry screening on the detection of duct dependent congenital heart disease: a Swedish prospective screening study in 39821 newborns. British Medical Journal 2009; 338: a3037.

https://doi.org/10.1136/bmj.a3037

PMid: 19131383 PMCid: PMC2627280

9. Guthrie R, Susi A. A simple phenylalanine method for detecting phenylketonuria in large populations of newborn infants. Pediatrics 1963; 32(3): 338-43.

PMid: 14063511

10. Wilson JMG, Jungner G and World Health Organization (1968). Principles and practice of screening for disease. Available from:

http://www.who.int/iris/handle/10665/37 650

11. Botto, L. D., Correa, A., \& Erickson, J. D. (2001). Racial and temporal variations in the prevalence of heart defects. Pediatrics, 107(3), e32-e32. https://doi.org/10.1542/peds.107.3.e32

12. Miniño AM, Heron MP, Smith BL. Deaths: preliminary data for
2004. National Vital Statistics Reports 2006; 54(19): 1-49.

13. Rosano A, Botto LD, Botting B, Mastroiacovo P. Infant mortality and congenital anomalies from 1950 to 1994 : an international perspective. Journal of Epidemiology \& Community Health 2000; 54(9): 660-6.

https://doi.org/10.1136/jech.54.9.660

PMCid: PMC1731756

14. van der Linde D, Konings EE, Slager MA, Witsenburg M, Helbing WA, Takkenberg $\mathrm{JJ}$, et al. Birth prevalence of congenital heart disease worldwide: a systematic review and meta-analysis. Journal of the American College of Cardiology 2011; 58(21): 2241-7.

https://doi.org/10.1016/j.jacc.2011.08.025 PMid: 22078432

15. Liske MR, Greeley CS, Law DJ, Reich JD, Morrow WR, Baldwin HS, et al. Report of the Tennessee task force on screening newborn infants for critical congenital heart disease. Pediatrics 2006; 118(4), e1250-e1256.

https://doi.org/10.1542/peds.2005-3061

PMid: 17015513

16. Mahle WT, Newburger JW, Matherne GP. Council on Cardiovascular Nursing, and Interdisciplinary Council on Quality of Care and Outcomes Research; American Academy of Pediatrics Section on Cardiology and Cardiac Surgery, and Committee on Fetus and Newborn. Role of pulse oximetry in examining newborns for congenital heart disease: a scientific statement from the American Heart Association and American Academy of Pediatrics. Circulation 2009; 120(5): 44758.

https://doi.org/10.1161/CIRCULATION

AHA.109.192576

PMid: 19581492

17. Sebelius K. Secretary of Health \& Human Services letter to the Secretary's Advisory Committee on Heritable Disorders in Newborns and Children (SACHDNC). 2011.

18. Ewer AK. 2013 Pulse oximetry screening for critical congenital heart defects in newborn infants: Should it be routine? Archives of Disease in Childhood Fetal and Neonatal Edition, August 2013; 99(1). 
19. Wren C, Reinhardt Z, Khawaja K. Twenty-year trends in diagnosis of lifethreatening neonatal cardiovascular malformations. Archives of Disease in Childhood Fetal and Neonatal Edition 2008; 93(1): F33-F35.

https://doi.org/10.1136/adc.2007.119032

PMid: 17556383

20. Kuehl KS, Loffredo CA, Ferencz C. Failure to diagnose congenital heart disease in infancy. Pediatrics 1999; 103(4): 743-7.

https://doi.org/10.1542/peds.103.4.743

PMid: 10103296

21. Thangaratinam S, Brown $\mathrm{K}$, Zamora J, Khan KS, Ewer AK. Pulse oximetry screening for critical congenital heart defects in asymptomatic newborn babies: a systematic review and metaanalysis. The Lancet 2012; 379(9835): 2459-64.

https://doi.org/10.1016/S01406736(12)60 107-X
22. Kochilas LK, Lohr JL, Bruhn E, BormanShoap E, Gams BL, Pylipow M, et al. Implementation of critical congenital heart disease screening

Minnesota. Pediatrics 2013; 132(3): e587-e594.

https://doi.org/10.1542/peds.2013-0803

PMid: 23958775 